\title{
Molecular crystals based on 9,10-distyrylanthracene derivatives with high solid state fluorescence efficiency and uniaxial orientation induced by supramolecular interactions
}

\author{
CHEN JinLong, MA SuQian, XU Bin ", ZHANG JiBo, DONG YuJie \& TIAN WenJing* \\ State Key Laboratory of Supramolecular Structure and Materials, Jilin University, Changchun 130012, China
}

Received January 29, 2013; accepted April 9, 2013; published online June 8, 2013

\begin{abstract}
Two 9,10-distyrylanthracene (DSA) derivatives CNDSA and t-BUDSA were designed and synthesized, and their photophysical properties and crystal structures were investigated. Compared to DSA, the maximum emission peaks of the two compounds showed red-shift not only in THF solution, but also in crystals, because the introduction of electron-withdrawing substituents to DSA leads to more dispersion of the electrons in the molecules. The two crystals of CNDSA and t-BUDSA show strong fluorescence with the efficiency $\Phi_{\mathrm{F}}$ of $45 \%$ and $33 \%$, respectively, which may be resulted from no obvious $\pi-\pi$ interactions between molecules inside the crystal due to the large distance between the two adjacent molecules and nearly no overlap between the central anthracene planes. The analysis of crystal structures of the two compounds indicated that the molecules are arranged in the same conformation and orientation in their crystals, i.e. uniaxially oriented packing crystal, because of the supramolecular interaction of $\mathrm{CH} / \pi$ in the two crystals and additional $\mathrm{C}-\mathrm{H} \cdots \mathrm{N}$ interactions in CNDSA.
\end{abstract}

9,10-distyrylanthracene derivatives, high solid state fluorescence efficiency, uniaxially oriented packing

Citation: $\quad$ Chen J L, Ma S Q, Xu B, et al. Molecular crystals based on 9,10-distyrylanthracene derivatives with high solid state fluorescence efficiency and uniaxial orientation induced by supramolecular interactions. Chin Sci Bull, 2013, 58: 2747-2752, doi: 10.1007/s11434-013-5897-9

Organic single crystals increasingly highlight their positions in material science and have attracted extensive attention [1-3] due to their unique properties, such as high thermostability and charge transport ability, low light propagation loss and reflecting mirror structure [4]. So they are prospectively applied in optical waveguides [5], solid-state laser [6], and many other high-tech areas [7,8]. As usual, molecular packing models can affect the fluorescence properties of organic crystals, as well as their practical applications. Among the packing models, uniaxially oriented packing is the most conductive to crystal optical properties, which is beneficial to the light propagation and stimulated emission due to the emission from the same orientation single molecules [4] During the last decade, only few works have reported on the uniaxially oriented molecular crystals [9], because it is difficult to make the organic molecules packing in the same

*Corresponding authors (email: wjtian@jlu.edu.cn; xubin@jlu.edu.cn) orientation due to the multiple conformation of organic molecules and the various interactions in the crystals [4]. Curtis and co-workers [9] reported two $\pi$-stack molecular crystals of alkyl bithiazole oligomers and the influence of intermolecular $(\pi-\pi)$ interactions on the spectra of conjugated molecules in the solid state. Ma and co-workers [4] reported a uniaxially oriented molecular crystal of cyano substituted oligo(p-phenyl enevinylene) (CNDPASDB) and its photophysical properties. Yan and co-workers [10] reported the uniaxially oriented crystals based on peptide. Another problem for organic crystal applications is that most fluorescent organic materials have strong fluorescence emissions in solution, but the emissions are generally weak in the solid-state $[11,12]$ attributed to high non-radiative decay rates by excitation energy trapping or strong intermolecular interactions [13-15]. Therefore, it will be of great importance in developing new organic single crystals with high solid state fluorescence efficiency and uniaxially 
oriented packing.

We have recently developed a series of 9,10-distyrylanthracene (DSA) derivatives, which can form high quality single crystals with high fluorescence efficiency [16]. According to their crystal structure analysis, intermolecular interactions in the crystals of these molecules not only induce the strong fluorescence emission, but also conduct the formation of the tight intermolecular stacking models. In addition, substitution of various groups in DSA is effective for modifying intermolecular interactions as well as forming different molecular packing feature $[16,17]$. However, there is almost no uniaxially oriented packing crystals have been got from DSA derivatives in previous works. Here, we introduced cyano-group $(\mathrm{CN})$ and tertiary butyl(t-BU) to DSA to synthesize two new molecules CNDSA and t-BUDSA in order to adjust intermolecular interactions and get uniaxially oriented packing crystals with high fluorescence efficiency. The investigation of their photophysical properties and analysis of their crystal structure indicate that the fluorescence efficiency are high and the packing model of CNDSA and t-BUDSA are uniaxially oriented, which is further demonstrating that introducing different substituent groups to DSA will lead to different intermolecular interactions, different fluorescence efficiency and different crystal packing models.

\section{Experimental}

Anthracene(99\%) was purchased from J\&K Chemical Co. (China). 4-tert-Butyibenzaldehyde and 4-cyanobenzaldehyde were purchased from Alfa Aesar Co.(China). Potassium tert-butoxide was purchased from Acros Organic Co. (Belgium). All other reagents were purchased as analytical grade from either Tianjin Fuyu Co.(China) or Beijing Chemical Reagent Co.(China) and used without further purification, unless otherwise noted. Anhydrous tetrahydrofuran(THF) was dried by distillation from sodium/benzophenone.

${ }^{1} \mathrm{H}$ NMR spectra were recorded on Bruker AVANVE $500 \mathrm{MHz}$ spectrometer with tetramethylsilane(TMS) as the internal standard. The time of flight mass spectra were recorded using a Kratos MALDI-TOF mass system.

UV-Vis absorption spectra were recorded on a Shimadzu UV-3100 spectrophotometer. Photoluminescence spectra were collected on a Shimadzu RF-5301PC spectrophotometer and Maya 2000Pro. optical fiber spectrophotometer. Crystalline state PL efficiencies were measured with an integrating sphere (C-701, Labsphere Inc. America), with a $405 \mathrm{~nm}$ Ocean Optics LLS-LED as the excitation source, and the laser was introduced into the sphere through the optical fiber.

Figure 1 shows the synthetic routes of CNDSA and tBUDSA. As the intermediate products, compound (1) 9,10bis(dichloromethyl)anthracene and compound (2) tetraethyl anthracene-9,10-diylbis(methylene)diphosphonate were synthesized according to the procedure reported [18].

\subsection{Synthesis of (CNDSA) 4,4'-(1E,1'E)-2,2'-(anthra- cene-9,10-diyl)bis(ethene-2,1-diyl) dibenzonitrile}

A round bottomed flask $(250 \mathrm{~mL})$ equipped with a magnetic stirring bar was charged with (2) tetraethyl anthracene9,10-diylbis(methylene)diphosphonate $(0.600 \mathrm{~g}, 1.25 \mathrm{mmol})$ and $\mathrm{t}-\mathrm{BuOK}(0.421 \mathrm{~g}, 3.76 \mathrm{mmol})$. The flask was then evacuated and charged with nitrogen. To the flask was added dropwise a solution of 4-cyanobenzal-dehyde $(0.345 \mathrm{~g}, 2.63$ $\mathrm{mmol})$ in THF $(70 \mathrm{~mL})$ at $0^{\circ} \mathrm{C}$ in an ice-water bath. The resulting mixture was stirred at room temperature for $12 \mathrm{~h}$. After removing the organic solvents (THF) with a rotary evaporator, the resultant precipitate was washed successively with $\mathrm{MeOH}$ and filtered off to give compound as

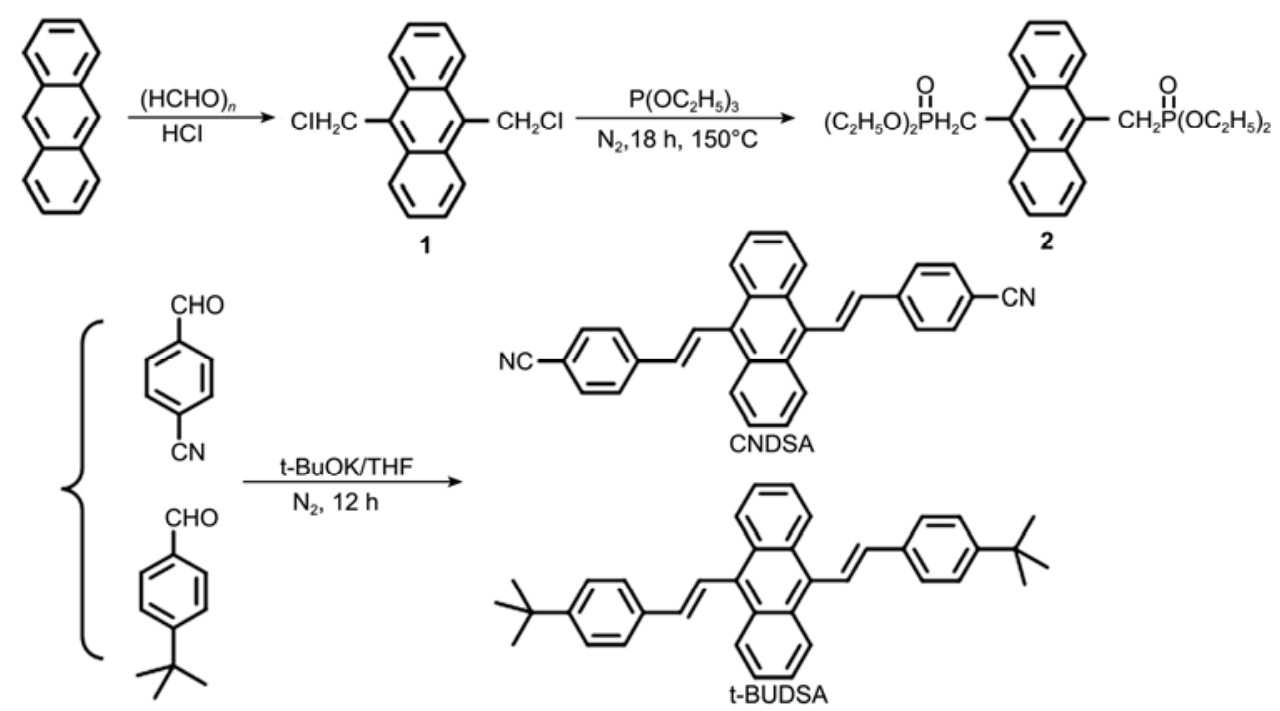

Figure 1 Synthetic routes for preparation of CNDSA and BUDSA. 
orange-yellow powder (60\% yield). ${ }^{1} \mathrm{H}$ NMR (500 MHz, $\left.\mathrm{CDCl}_{3}\right): \delta 8.325-8.346(\mathrm{~m}, 4 \mathrm{H}, \mathrm{Ar}), 8.067-8.100(\mathrm{~d}, J=$ $16.5 \mathrm{~Hz}, 2 \mathrm{H}, \mathrm{CH}=\mathrm{CH}), 7.742-7.784(\mathrm{~m}, 8 \mathrm{H}, \mathrm{Ar}), 7.510-$ 7.530 (m, 4H, Ar), 6.959-6.992 (d, J=16.5 Hz, 2H, CH= $\mathrm{CH})$. MALDI/TOF MS calcd. for $\mathrm{C}_{32} \mathrm{H}_{20} \mathrm{~N}_{2}: 432.51$. Found: 432.24. Anal. calcd. for $\mathrm{C}_{32} \mathrm{H}_{20} \mathrm{~N}_{2}$ : C, 88.66; $\mathrm{H}, 4.66 ; \mathrm{N}$, 6.48. Found: C, 88.68; H, 4.67; N, 6.44 .

\subsection{Synthesis of (t-BUDSA) 9,10-bis(4-tert-butylstyryl)- anthracene}

A round bottomed flask $(250 \mathrm{~mL})$ equipped with a magnetic stirring bar was charged with $2(0.600 \mathrm{~g}, 1.25 \mathrm{mmol})$ and t-BuOK $(0.421 \mathrm{~g}, 3.76 \mathrm{mmol})$. The flask was then evacuated and charged with nitrogen. To the flask was added dropwise a solution of 4-tert-butyibenzaldehyde $(0.426 \mathrm{~g}, 2.63 \mathrm{mmol}$, $0.439 \mathrm{~mL})$ in THF $(70 \mathrm{~mL})$ at $0^{\circ} \mathrm{C}$ in an ice-water bath. The resulting mixture was stirred at room temperature for $12 \mathrm{~h}$. After removing the organic solvents (THF) with a rotary evaporator, the resultant precipitate was washed successively with $\mathrm{MeOH}$ and filtered off to give compound as orangeyellow powder (55\% yield). ${ }^{1} \mathrm{H}$ NMR $\left(500 \mathrm{MHz}, \mathrm{CDCl}_{3}\right): \delta$ 8.386-8.405 (m, 4H, Ar), 7.889-7.922 (d, J=16.5 Hz, 2H, $\mathrm{CH}=\mathrm{CH}), 7.641-7.657$ (d, 4H, Ar), 7.492-7.508 (d, 4H, Ar), 7.445-7.465 (m, 4H, Ar), 6.907-6.940 (d, J=16.5 Hz, 2H, $\mathrm{CH}=\mathrm{CH}), 1.396$ (s, 18H, $\left.\mathrm{CH}_{3}\right)$. MALDI/TOF MS calcd. for $\mathrm{C}_{38} \mathrm{H}_{38}$ : 494.71. Found: 494.31. Anal. calcd. for $\mathrm{C}_{38} \mathrm{H}_{38}$ : C, 92.26; H, 7.74. Found: C, 92.30; H, 7.72.

\section{Results and discussion}

The photophysical properties of the two compounds were investigated by UV-Vis absorption spectroscopy and photoluminescence (PL) spectroscopy in THF solution and solid state. Figure 2(a) and (b) show the UV and PL spectra of CNDSA and t-BUDSA, respectively and Table 1 summarizes the related results of the two molecules. From Figure 2, we know that the maximum absorptions of CNDSA and t-BUDSA appear at 418 and $410 \mathrm{~nm}$ similar to that of DSA $(414 \mathrm{~nm})$ [16], which are originated from the absorption of distyrylanthracene entity and little affected by the peripheral substituent [19]. In addition, both of CNDSA and t-BUDSA exhibited weak and orange emission with maximum PL peaks at 625 and $615 \mathrm{~nm}$ in THF solution. Compared to DSA $(612 \mathrm{~nm})$, CNDSA showed a red-shift of $13 \mathrm{~nm}$ because the introduction of electron-withdrawing substituent $(\mathrm{CN})$ to DSA can lead to more dispersion of the electrons and more extended $\pi$-conjugation in the molecules, which can also be demonstrated by theoretical calculations (Figure 3) [20].

The PL spectra of CNDSA and t-BUDSA single crystals are also shown in Figure 2. CNDSA exhibited orange emission with maximum peak at $582 \mathrm{~nm}$ and t-BUDSA exhibited green emission with maximum peak at $530 \mathrm{~nm}$. Compared to DSA (516 nm), CNDSA showed red-shift of $66 \mathrm{~nm}$ while
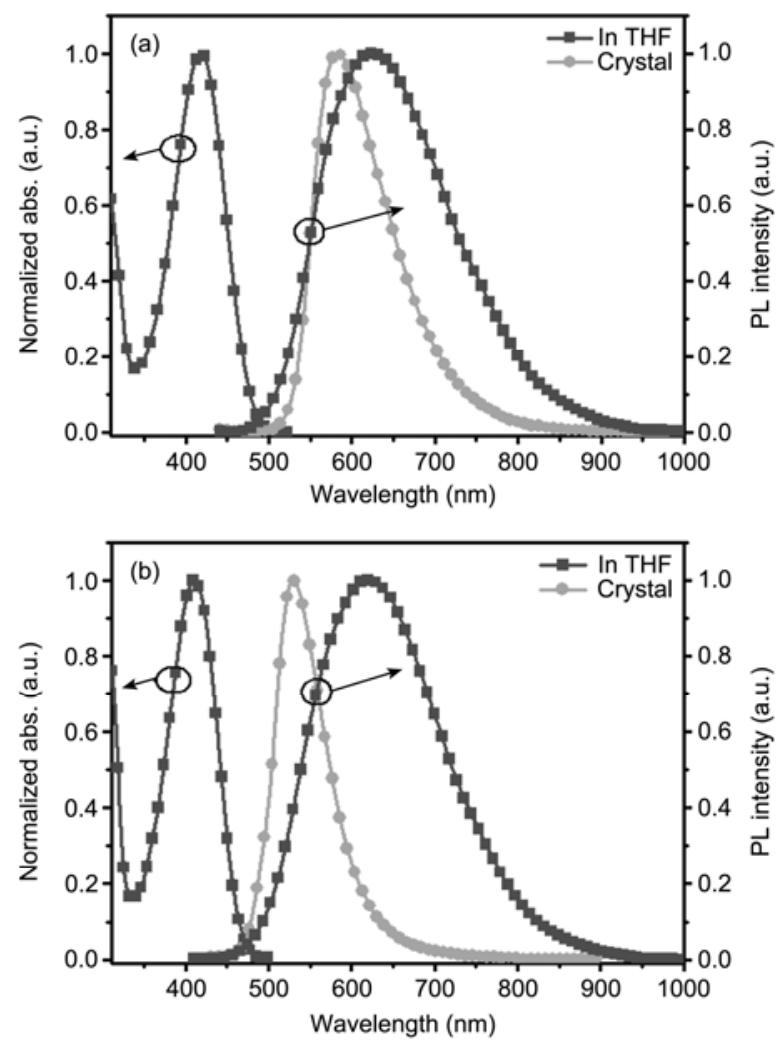

Figure 2 Normalized UV-Vis absorption and PL spectra of CNDSA (a) and t-BUDSA (b).

Table 1 Optical properties of CNDSA and t-BUDSA

\begin{tabular}{lccccc}
\hline Compound & $\mathrm{Abs}_{(\mathrm{THF})}$ & $\mathrm{PL}_{(\mathrm{THF})}$ & $\mathrm{PL}_{(\mathrm{Cry})}$ & $\Phi_{\mathrm{F}(\mathrm{THF})}$ & $\Phi_{\mathrm{F}(\mathrm{Cry})}$ \\
\hline CNDSA & 418 & 625 & 582 & $25 \%$ & $45 \%$ \\
t-BUDSA & 410 & 615 & 530 & $35 \%$ & $33 \%$ \\
\hline
\end{tabular}

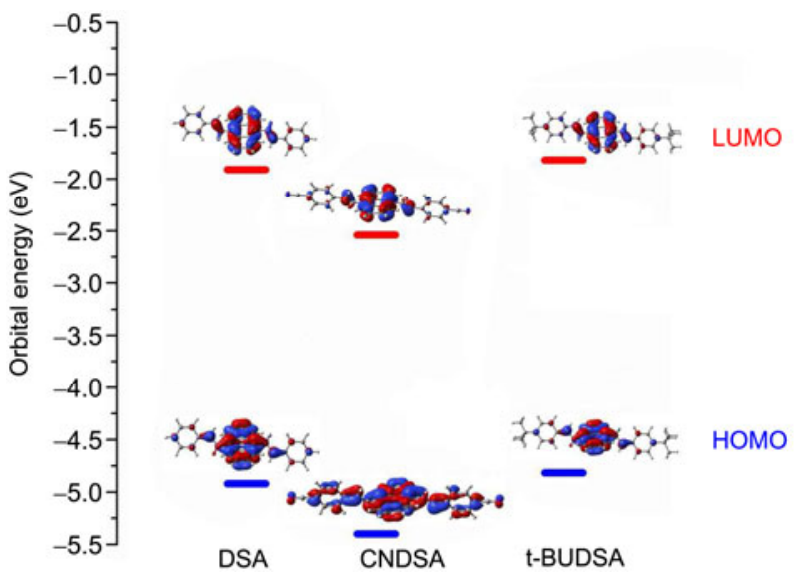

Figure 3 The frontier molecular orbitals for DSA, CNDSA and t-BUDSA.

the red-shift of t-BUDSA is $14 \mathrm{~nm}$ due to the introduction of electron-withdrawing substituent. The large red-shift of CNDSA resulted from the stronger electronic withdrawing 
ability of $\mathrm{CN}$ than that of t-BU. Compared to the PL spectra of the two compounds in THF solution, CNDSA and tBUDSA crystals exhibit 43 and $85 \mathrm{~nm}$ blue-shifts because of large distorted conformation of the two compounds in the crystal states, which will be demonstrated by the further analysis of the crystal structure. Moreover, both of CNDSA and t-BUDSA exhibited strong fluorescence emission in their crystals and the fluorescence efficiency $\Phi_{\mathrm{F}}$ are $45 \%$ and $33 \%$, respectively. From our previous studies [13,16,17], we know the crystal fluorescence efficiency may be related to the intermolecular interactions inside crystal [21]. In order to further understand the relationship between the crystal intermolecular interactions and crystal fluorescence efficiency, both of the crystal packing models and intermolecular interactions were investigated.

Single crystals of CNDSA and t-BUDSA were prepared by vaporizing mixed solvents of chloroform and ethanol slowly at room temperature. The unit cells of CNDSA and t-BUDSA are both triclinic, space group $P-1$, containing one discrete molecule. The ball-and-stick model structures and crystal photos of CNDSA and t-BUDSA are shown in Figure 4. Similar to DSA single crystal [16], CNDSA and t-BUDSA molecules in crystal are remarkably twisted. The torsional angles of the double bonds $\theta(2,3,4,5)$ is $179.9^{\circ}$ in the CNDSA crystal and $179.3^{\circ}$ in the t-BUDSA (Table 2), larger than that of DSA $\left(172.2^{\circ}\right)$, resulting in relatively less twisted molecular structure. The torsional angles $\theta(1,2,3,4)$ of CNDSA and t-BUDSA are $54.4^{\circ}$ and $60.7^{\circ}$, larger than that of DSA $\left(4.6^{\circ}\right)$ and $\theta(3,4,5,6)$ between the double bonds and the adjacent phenyl ring are $20.6^{\circ}$ for CNDSA and $4.6^{\circ}$ for t-BUDSA, smaller than that of DSA $\left(75.0^{\circ}\right)$ due to the effect of internal steric hindrance between the vinylene moieties and the substituents moieties ( $\mathrm{CN}$ and t-BU). The analyses demonstrate that the two compounds have nonplanar conformation in their crystals.

Interestingly, CNDSA and t-BUDSA are found to array parallel with the identical conformation and orientation in their crystals (Figures 5(a) and 6(a)), i.e., so-called uniaxially oriented crystals. According to meticulous analysis of crystal
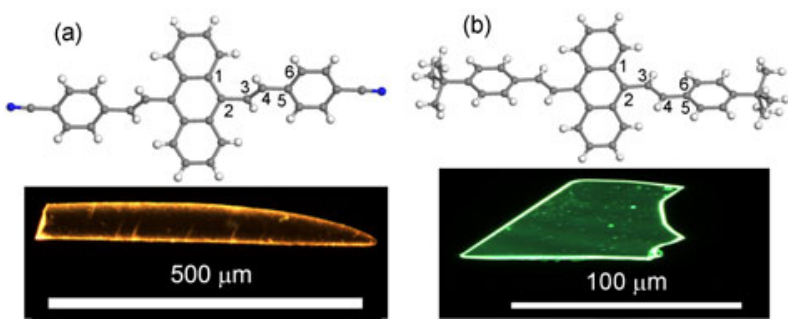

Figure 4 The ball-and-stick model structures and crystal photos of CNDSA (a) and t-BUDSA (b)

Table 2 Selected dihedral angles (deg) in crystal structures of CNDSA and t-BUDSA

\begin{tabular}{lccc}
\hline Compound & $\theta_{(1,2,3,4)}$ & $\theta_{(2,3,4,5)}$ & $\theta_{(3,4,5,6)}$ \\
\hline CNDSA & 54.4 & 179.9 & 20.6 \\
t-BUDSA & 60.7 & 179.3 & 4.6 \\
\hline
\end{tabular}

structures, we found it is the supramolecular interactions that induce CNDSA and t-BUDSA to assemble themselves into the absolute uniaxial orientation. In the CNDSA crystal, as shown in Figure 5(b), a $\mathrm{CH} / \pi$ interaction (I) $[22,23]$ is formed between two molecules with an interaction distance of $2.79 \AA$ and an angle of $143.8^{\circ}$, where the vinylene (Vin) moiety along the long axis of one molecule acts as an $\mathrm{H}$ donor and the corresponding anthrylene core of the adjacent molecule acts as an $\mathrm{H}$ acceptor. Hence, the $\mathrm{CH} / \pi$ intermolecular interactions connect CNDSA molecules to construct the molecular clusters, in which each CNDSA molecule preserves the same orientation.

Besides, two types of $\mathrm{C}-\mathrm{H} \cdots \mathrm{N}$ interactions $[23,24] \mathrm{II}$ and III exist in the crystal (Figure 5(c)). The $\mathrm{H}^{\cdots \cdots \mathrm{N}}$ distance of two interactions II and III are 2.75 and 2.79, and the angles are $146.0^{\circ}$ and $142.5^{\circ}$, respectively. The $\mathrm{C}-\mathrm{H} \cdots \mathrm{N}$ interactions (II and III) attach the molecular clusters and maintain the same orientation of CNDSA molecules along the distyrylanthracene direction.

In t-BUDSA crystal, there are two aromatic $\mathrm{CH} / \pi$

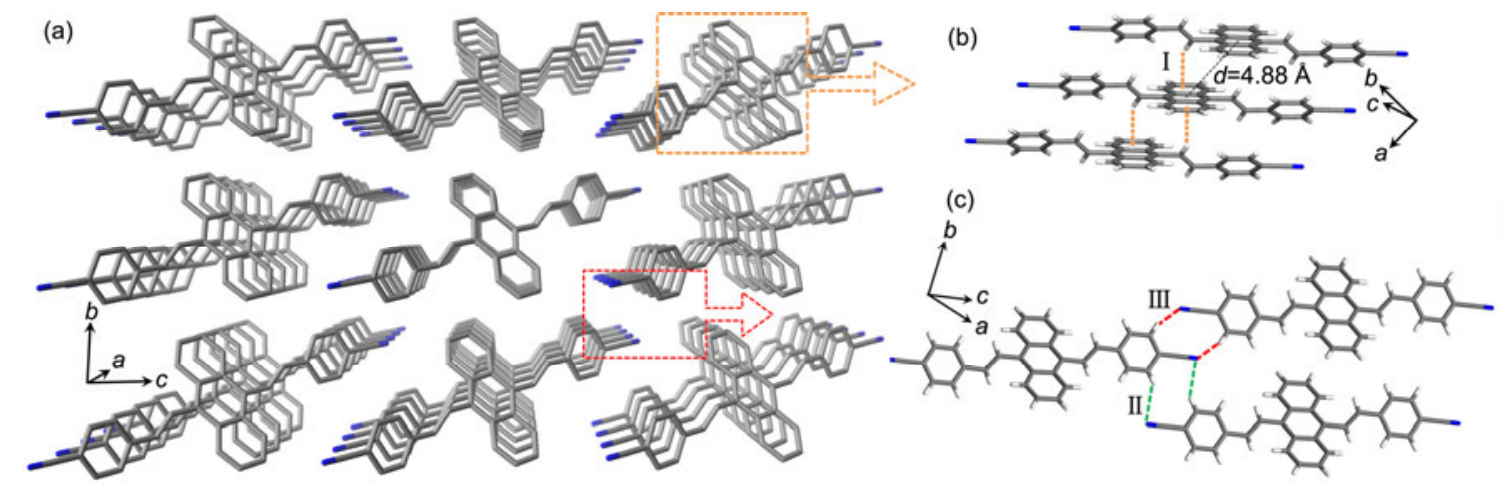

Figure 5 (a) Uniaxially oriented alignment of CNDSA molecules in the crystal. (b) $\mathrm{CH} / \pi$ interactions I and the distance between two molecules $d$. (c) $\mathrm{C}-\mathrm{H} \cdots \mathrm{N}$ interactions II and III. 


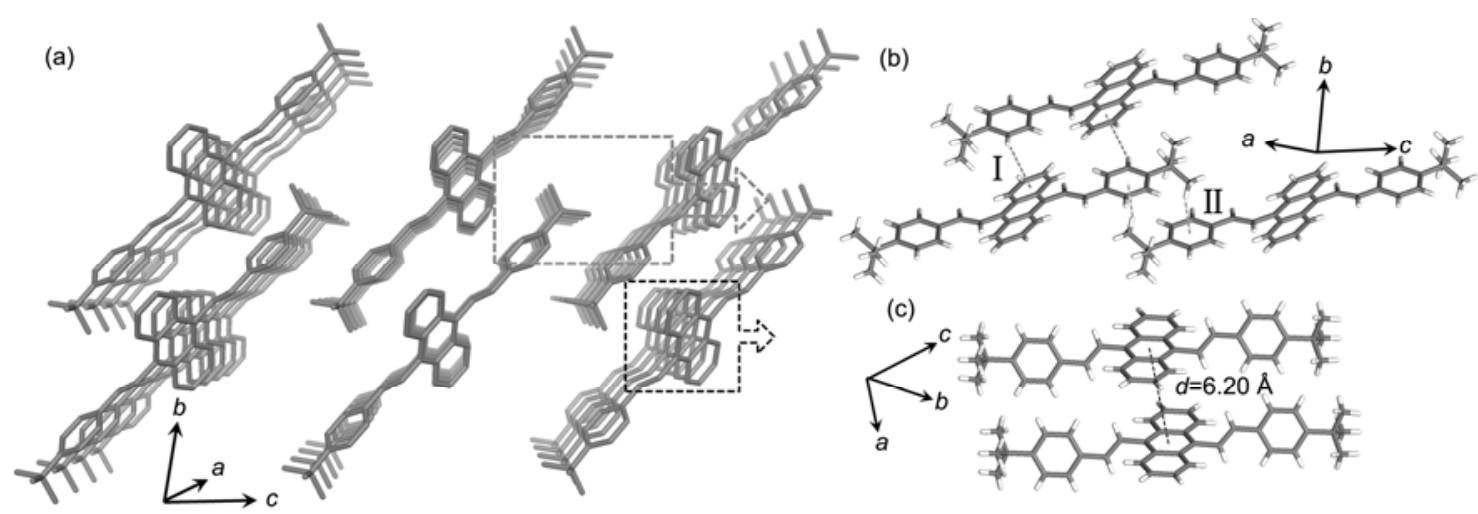

Figure 6 (a) Uniaxially oriented alignment of t-BUDSA molecules in the crystal. (b) $\mathrm{CH} / \pi$ interactions I and II. (c) the distance between two molecules $d$.

Table 3 Summary of the $\mathrm{CH} / \pi$ and $\mathrm{C}-\mathrm{H} \cdots \mathrm{N}$ interactions in the two crystals

\begin{tabular}{cccc}
\hline Compound & Orientation of interaction & $d(\AA)$ & $A\left(^{\circ}\right)$ \\
\hline CNDSA(Figure 4) & I Vin $\rightarrow$ An & 2.79 & 143.8 \\
& II C-H $\cdots \mathrm{N}$ & 2.75 & 146.0 \\
& III C-H $\cdots \mathrm{N}$ & 2.79 & 142.5 \\
t-BUDSA(Figure 5) & I CH $\rightarrow$ Ph & 3.02 & 130.1 \\
& II Ph $\rightarrow$ An & 3.13 & 151.0 \\
\hline
\end{tabular}

interactions (I and II) as shown in Figure 6(b). For interaction I, formed between the phenyl ring and the anthrylene core, the distance and angle of $\mathrm{C}-\mathrm{H}-\pi$ center are $3.02 \AA$ and $130.1^{\circ}$. Interaction II is formed between phenyl ring of one molecule and tert-butyl group of the adjacent molecule, and the interaction distance and angle of $\mathrm{C}-\mathrm{H}-\pi$ center are 3.13 $\AA$ and $151.0^{\circ}$. The $\mathrm{CH} / \pi$ interaction I (Figure 6(b)) attach the molecular clusters and conduct the uniform orientation of t-BUDSA molecules, and the interaction II (Figure 6(b)) connect the adjacent clusters to form uniaxial orientation crystal. Compared to the interactions in DSA crystal, the formed $\mathrm{C}-\mathrm{H} \cdots \mathrm{N}$ interactions II and III (Figure 5(c)) for $\mathrm{CNDSA}$ and $\mathrm{CH} / \pi$ interaction II (Figure $6(\mathrm{~b})$ ) for t-BUDSA reduce the twisting angle $\theta(3,4,5,6)$ between double bond and the anthracene plane, as shown in Table 2, which results in a relatively less twisted and rigid molecular structure, avoiding free rotation movement in the crystal and inducing a tight uniaxially oriented intermolecular packing. According to the punctilious analysis, there is no obvious $\pi-\pi$ interactions formed in the crystals due to the large distance between the anthracene core of the two adjacent molecules (4.88 $\AA$ for CNDSA and 6.20 $\AA$ for t-BUDSA, Figures 5(b) and $6(\mathrm{c})$ ) and there is nearly no overlap between the central anthracene planes, which is beneficial to inducing high crystal fluorescence efficiency.

\section{Conclusions}

In this study, we reported two new DSA derivatives CNDSA and t-BUDSA. Both of them showed high solid state fluores- cence efficiency (45\% for CNDSA and 33\% for t-BUDSA) because there is no obvious $\pi-\pi$ interactions formed in their crystals. Furthermore, CNDSA and t-BUDSA are both uniaxially oriented crystal due to the $\mathrm{CH} / \pi$ supramolecular interaction in the two crystals and additional $\mathrm{C}-\mathrm{H} \cdots \mathrm{N}$ interactions in CNDSA. These characteristics of the two compounds indicate that they may be applied in organic optoelectronic field.

This work was supported by the National Basic Research Program of China (2013CB834702), the National Natural Science Foundation of China $(21074045,21204027,21221063)$, the Research Fund for the Doctoral Program of Higher Education of China, and the Project of Jilin Province (20100704).

1 Yanagi H, Ohara T, Morikawa T. Self-waveguided gain-narrowing of blue light emission from epitaxially oriented p-sexiphenyl crystals. Adv Mater, 2001, 13: 1452-1455

2 Tok J B H, Bao Z A. Recent advances in flexible and stretchable electronics, sensors and power sources. Sci China-Chem, 2012, 55: $718-725$

3 Wang J H, Liu L, Liu G F, et al. Three novel photoisomeric compounds of the 4-acyl pyrazolone derivants: Crystal structures and substituent effects on photo-isomerism in solid state. Sci China Ser BChem, 2008, 51: 661-668

4 Li Y P, Shen F Z, Wang H, et al. Supramolecular network conducting the formation of uniaxially oriented molecular crystal of cyano substituted oligo( $p$-phenylenevinylene) and its amplified spontaneous emission (ASE) behavior. Chem Mater, 2008, 20: 7312-7318

5 Cáceres D, Cebrián C, Rodríguez A M, et al. Optical waveguides from 4-aryl-4H-1,2,4-triazole-based supramolecular structures. Chem Commun, 2013, 49: 621-623

6 Park S, Kwon O H, Kim S, et al. Imidazole-based excited-state intramolecular proton-transfermaterials: Synthesis and amplified spontaneous emission from a large single crystal. J Am Chem Soc, 2005, 127: 10070-10074

7 Yanagi H, Yoshiki A. Stimulated resonance Raman scattering from epitaxially oriented crystals of biphenyl-capped thiophene. Appl Phys Lett, 2004, 84: 4783-4785

8 Varghese S, Yoon S J, Calzado E M, et al. Stimulated resonance Raman scattering and laser oscillation in highly emissive distyrylbenzene-based molecular crystals. Adv Mater, 2012, 24: 6473-6478

9 Koren A B, Curtis M D, Francis A H, et al. Intermolecular interactions in $\pi$-stacked conjugated molecules. Synthesis, structure, and spectral characterization of alkyl bithiazole oligomers. J Am Chem Soc, 2003, 125: 5040-5050

10 Yan X H, Su Y, Li J B, et al. Uniaxially oriented peptide crystals for 
active optical waveguiding. Angew Chem Int Ed, 2011, 50: 1118611191

11 Xu B, He J T, Dong Y J. Aggregation emission properties and selfassembly of conjugated oligocarbazoles. Chem Commun, 2011, 47: 6602-6604

12 Gierschner J, Ehni M, Egelhaaf H J, et al. Solid-state optical properties of linear polyconjugated molecules: $\pi$-stack contra herringbone. $\mathrm{J}$ Chem Phys, 2005, 123: 144914-144922

13 Xu B, Fang H H, Dong Y J, et al. Solid state emission enhancement of 9,10-distyrylanthracene derivatives and amplified spontaneous emission from a large single crystal. New J Chem, 2010, 34: 18381842

14 Qin A J, Zhang Y, Han N, et al. Preparation and self-assembly of amphiphilic polymer with aggregation-induced emission characteristics. Sci China-Chem, 2012, 55: 772-778

15 Xue Y N, Chai S Y, Bie G J, et al. Synthesis and properties of a novel aggregation-induced emitter 9,10-bis[2-(1-methyl-1H-pyrrol-2-yl) vinyl]anthracene (in Chinese). Acta Chim Sinica, 2008, 66: 15771582

16 He J T, Xu B, Chen F P, et al. Aggregation-induced emission in the crystals of 9,10-distyryl anthracene derivatives: The essential role of restricted intramolecular torsion. J Phys Chem C, 2009, 113: 9892-9899

17 Dong Y J, Xu B, Zhang J B, et al. Supramolecular interactions induced fluorescent organic nanowires with high quantum yield based on 9,10-distyrylanthracene. Cryst Eng Comm, 2012, 14: 6593-6598

18 Dong Y J, Xu B, Zhang J B, et al. Piezochromic luminescence based on the molecular aggregation of 9,10-bis((E)-2-(pyrid-2-yl)vinyl) anthracene. Angew Chem Int Ed, 2012, 51: 10782-10785

19 Dong Y J. Small molecules based on divinylanthracene: Design and synthesis, crystal structure, self-assembly and piezochromic properties (in Chinese). Dissertation for Master Degree. Changchun: Jilin University, 2012

20 Wang L J, Xu B, Zhang J B, et al. Theoretical investigation of electronic structure and charge transport property of 9, 10-distyrylanthracene (DSA) derivatives with high solid-state luminescent efficiency. Phys Chem Chem Phys, 2013, 15: 2449-2458

21 Jenning W B, Farrell B M, Malone J F. Attractive intramolecularedge-to-face aromatic interactions in flexible organic molecules. Acc Chem Res, 2001, 34: 885-894

22 Weiss $\mathrm{H} \mathrm{C}$, Bläser $\mathrm{D}$, Boese $\mathrm{R}$, et al. $\mathrm{C}-\mathrm{H}^{\cdots} \cdots \pi$ interactions in ethynylbenzenes: The crystal structures of ethynylbenzene and 1,3,5triethynylbenzene, and a redetermination of the structure of 1,4-diethynylbenzene. Chem Commun, 1997, 1703-1704

23 Steiner T. The hydrogen bond in the solid state. Angew Chem Int Ed, 2002, 41: 48-76

24 Taylor R, Kennard O. Crystallographic evidence for the existence of C-H-O, C-H-N, and C-H-Cl hydrogen bonds. J Am Chem Soc, 1982, 104: 5063-5070

Open Access This article is distributed under the terms of the Creative Commons Attribution License which permits any use, distribution, and reproduction in any medium, provided the original author(s) and source are credited. 